\title{
Mathematics, mathematical physics and developing countries
}

It is not self-evident that mathematics and perhaps, even more, mathematical physics can be useful to the establishment and growth of scientific communities in developing countries. Not everyone will agree that they do have a role to play and though many of the ideas presented here have been discussed with friends, they are essentially the author's own views based on what is recognized to be a quite subjective analysis.

\section{Need for a Scientific Community}

A strong scientific community exists in all the developed countries and there is no doubt that it has contributed greatly to the general welfare of such countries. It is generally accepted that it could also significantly help less favoured countries and in two ways. First, by providing skills, knowledge and experience where they are needed and second, by allowing the formation and training of the younger generation directly in the countries where they will have to work.

The first point is clear as soon as it is appreciated that the most important factor in the brain drain away from the developing countries, is that their scientists and doctors, trained at high cost in industrialized countries, find themselves completely isolated when they return home. Recognition of this fact was incidentally a prime motivation for the creation of the International Centre for Theoretical Physics in Trieste, whose purpose is to enable physicists from developing countries, periodically to spend a few months re-establishing contact with the main stream of research.

The second point is even more obvious. Teaching and training young people is cheaper and can be more specifically aimed if it is done in the developing countries themselves. As for the cost, it is clear that even if one has to offer a very attractive salary to a teacher to have him come to the country, it is still much cheaper than sending the students abroad, at least so long as the teacher does not need equipment. Moreover, a large fraction of the salary of the teacher will be spent where he lives, which is more favourable to the balance of payments than sending students away. At the same time, not sending the younger people abroad for too long considerably reduces the danger of the brain drain. What developing countries need most and fast, is a very large number of very competent primary and secondary school teachers, technicians and engineers on the spot. So many can only be educated in the country itself, if there exists there a valid scientific community. It is an elementary point but one which must be repeated again and again, that the value of the teaching, crucially depends upon the value of the teacher.

\section{Establishing a Scientific Community}

The question of how to establish and maintain a strong scientific community is of course very complex. We shall simplify it by concentrating on three factors which we think are decisive in making a scientist want to come and stay at a given place. These factors we describe by the catchwords Finances, Geography, Science, Under finances, we group the elements that go to make up the standard of living which can be offered to the scientist. It is an aspect that cannot be characterized by a universal figure, because cost of living, fringe benefits, etc. vary considerably from place to place. Under geography we bring together all the factors connected with the actual location of the place in question, such as educational facilities for the children, the cultural life, climate, recreation possibilities, historical or artistic surrounding, facilities for communications etc. Finally under science, we have all the factors which influence scientific life : number and level of colleagues and the collaboration that is to be found, technical facilities and equipment, the teaching load and so on.

Even a superficial observation of the evolution of the existing research and teaching centres, shows that in order to achieve a reasonably rapid and stable development, it is desirable that all three factors should be positive. Even if only two are adequately covered, something can still be achieved; but if there is only one good point, it had better be the science. In any case, the science side is usually the only one which can be modified by scientists, so it seems pertinent to ask where to start, especially if the means are not unlimited.

\section{Guenin, Geneva}

We make the asumption that it is easier to attract somebody of high level as the first in a given domain, if you have already got very good people in neighbouring fields. This assumption is not necessarily always true but should apply in a majority of cases.

\section{What is Mathematical Physics}

So far, nothing of what we have said seems to imply that mathematical physics can be of any use in resolving the problems posed, and for most physicists, mathematical physics is just one of many marginal fields of physics. That is a fair point of view if the parameter used in the assessment is the number of people involved and also if only a restrictive definition is employed. In the broadest sense, mathematical physics is that part of theoretical physics which is not purely phenomenological, together with the part of mathematics which is devoted to the study of the abstract structures of physics. It does not comprise computational physics. Even if such an embracing definition is not considered acceptable, the marginality of mathematical physics appears completely different from that of other domains, because every problem of physics embodies an aspect which fits into the framework of mathematical physics. In a sense, mathematical physics would be better called fundamental physics, if only every branch of physics were not, in fact, fundamental. So we shall keep the nomenclature mathematical physics for lack of a better one.

\section{Mathematical Physics in Teaching Programmes}

It has not infrequently been said that one should teach people mainly things which lie outside what they are going to do, because what they will need for their job they will have to learn anyway, whereas once studies are terminated (with very few exceptions), they are not going to acquire much knowledge outside their specialized field. To some this may sound a little like a joke, but if not pushed to its extreme it contains in our opinion a certain amount of truth. That is why personally I have always insisted on teaching future experimentalists and phenomenologists a sub- 
stantial amount of mathematics and abstract theoretical physics, and on sending future theoreticians into the laboratory. With the exception of those who later will be doing research in mathematical physics, for most, mathematics and abstract theoretical physics is the baggage for life that they assembled by the time they got their diploma. This abstract formation has the disadvantage that the people who received it are less immediately efficient than those which will already have practised a lot in their future field of specialization, but it has at least the tremendous advantage of being much more time resistant. Mathematical theorems remain true even after twenty years and someone who has got into the habit of discerning the basic structures of existing theories will have a much easier time recycling himself, because he will be able to extract the basic features of the theories which are going to emerge in his subsequent work.

If it is agreed that an abstract and mathematical formation becomes obsolete more slowly than other training, the question may then be asked: why not only pure mathematics? This could be envisaged, but there is a big difference between pure mathematics and mathematical physics, and that difference lies in Nature. The abstract theories of physics are not allowed to be only pure constructions of the mind, like mathematics. They ultimately must represent a model of the world, or at least some classes of phenomena which, up to a given approximation can be fitted to experiment. Mathematics is, in any case, a must for every education. It belongs to culture in exactly the same way as the ninth symphony, or Hamlet but it is, in addition, the language of science. Mathematical physics on the other hand cannot be taught at all levels. Only for future mathematicians and physicists (whatever their future field of specialization might be), can it be allowed to become a dominant part of the curriculum.

\section{Mathematical Physics and Creation of Scientific Communities}

We now come, to the reasons why we believe that mathematical physics can make a useful contribution to the creation and development of scientific communities in less favoured regions of the world. There is, first, a question of "market". Mathematical physics is not fashionable, and the number of people working in the field is small; a few hundreds only are really active in the whole world. That means that there is a great deal to be done and new-comers have a fair chance of reaching really international level. The second point is that those who are working very far from the main research centres, are not placed at any real disadvantage. All that they need is a decent library and a good mail system. If furthermore they can attend one conference or school in the year, they will not feel discriminated against in respect to others. The situation is very different for phenomenologists. The field is very crowded, and you have no chance, if you are not in direct touch with the big laboratories, of being aware of any development before it actually appears in the literature. A third point is the relative cheapness of the investments needed. A first class library can be obtained at a total cost of about one million dollars and its yearly running costs for acquisitions are of the order of magnitude of 50,000 dollars. Such a library can also be used with very little supplements for the whole field of physics. The rest of the costs are only salaries and building and maintenance of simple office rooms. Big computers are not an absolute must, even if they sometimes can be useful. Apart from mathematics, one cannot have any cheaper science. Finally, one has to realize that mathematical physics is a good departure point for further developments. There is a continuous passage from the most abstract mathematical consideration to very concrete phenomenological study and also to definitely applied problems. An extension to neighbouring fields is therefore relatively easy to perform, especially if there are highly competent people who are continuing in the more abstract domains.

\section{Conclusions}

We believe that these arguments are sufficiently strong to make out the case that mathematical physics can be useful in the development of science, especially where it has not yet established itself. It has nevertheless to be stated that this is not an attempt to position it at the top of some scale of values. We do not think that mathematical physics is in any way more important or more interesting than other ways of looking at physical problems. We only think that it is a convenient place to make a start; but to stop there would, of course, be a pity.

\section{Conference Report}

\section{Pluridimensional Radiative Transfer}

The solar atmosphere shows convective and magnetic structure on scales down to the observational resolution $(\approx 200 \mathrm{~km})$. The radiative transport in the small structures, cannot be adequately treated by means of one dimensional radiative transfer in mean models, describing the thermodynamic and magnetogasdynamic parameters as a function of one spatial coordinate only. In recent years, several astrophysicists in Europe have become engaged in problems involving pluridimensional ra- diative transfer (PDRT) and the committee preparing the establishment of the Solar Physics Section of the Astronomy and Astrophysics Division, supported a plan to bring these people together in a workshop, i.e. an informal meeting of participants invited of rather restricted numbers upon application.

The Workshop was organized in the Osservatorio Astronomico di Capodimonte in Naples, 14-16 September, under the tittle: "Pluridimensional Radiation Transfer - Theory and
Application to Structures in Stellar Atmospheres". The meeting, sponsored by the EPS and supported by the Consiglio Nazionale della Recerche, was attended by 15 participants.

The Workshop was arranged in three stages:

1. Each participant summarized the PDRT problem which arose in his work by indicating the astrophysical background, the physical and geometrical simplifications, the mathematical methods, and results obtained and/or problems encountered. These 